\title{
The ERK2 Mitogen-Activated Protein Kinase Regulates the Timing of Oligodendrocyte Differentiation
}

\author{
Sharyl L. Fyffe-Maricich, J. Colleen Karlo, Gary E. Landreth, and Robert H. Miller \\ Department of Neurosciences, Case Western Reserve University, Cleveland, Ohio 44106
}

Oligodendrocyte development is tightly controlled by a variety of extracellular growth and differentiation factors. The mitogen-activated protein kinases (MAPKs), ERK1 and ERK2, are critical intracellular signaling molecules important for transducing these extracellular signals. The extracellular signal-regulated kinases (ERKs) are ubiquitously expressed, coordinately regulated, and highly similar, but Erk2 deletion in mice is embryonic lethal whereas Erk1 deletion is not. Several studies have suggested that MAPK signaling is important for oligodendrocyte differentiation, although specific roles for the two ERK isoforms have not been investigated. In this study, we deleted $E r k 2$ in the developing mouse cortex from GFAP-expressing radial glia that generate neurons and oligodendrocytes. In vitro analysis revealed that loss of ERK2 resulted in fewer galactocerebroside-expressing mature oligodendrocytes in cortical cultures. In vivo, a delay in the expression of the myelin protein MBP was observed in the corpus callosum at postnatal day 10 (P10). In contrast, Erk1 deletion did not affect oligodendrocyte differentiation. By P21, MBP expression was restored to wild-type levels, demonstrating that the loss of ERK2 results in a delay but not a complete arrest in the appearance of differentiated oligodendrocytes in vivo. Importantly, both the proliferation and total number of oligodendrocyte progenitor cells (OPCs) appeared normal in the Erk2 conditional knock-out cortex, demonstrating that ERK2 plays a specific role in the timing of forebrain myelination but is not critical for the proliferation or survival of OPCs. Oligodendrocyte-specific deletion of Erk2 also resulted in decreased levels of MBP, indicating a cell-autonomous effect of ERK2 in the oligodendrocyte lineage.

\section{Introduction}

Oligodendrocytes play a critical role in the brain and spinal cord by generating myelin sheaths that envelop axons providing the electrical insulation needed to maximize conduction velocity. The importance of oligodendrocytes and myelin becomes obvious in diseases such as multiple sclerosis in which demyelinated lesions result in devastating physical and cognitive deficits.

Throughout development, oligodendrocyte progenitor cells (OPCs) progress through a series of morphological and antigenic stages before maturing and producing myelin (Pfeiffer et al., 1993; Miller, 1996). The importance of extracellular signaling factors for oligodendrocyte differentiation is widely recognized, but little is known about the specific intracellular signaling pathways that transduce these signals. Platelet-derived growth factor (PDGF) and fibroblast growth factor-2 (FGF-2) are potently mitogenic to OPCs and play important roles in the timing of oligodendrocyte differentiation (Noble et al., 1988; Raff et al., 1988; McKinnon et al., 1990; Bansal and Pfeiffer, 1994). IGF-I and neurotrophins promote the differentiation and/or survival of OPCs (Mozell and McMorris, 1991; Barres et al., 1992; Kumar et

Received June 22, 2010; revised 0ct. 26, 2010; accepted Nov. 3, 2010.

This work was supported in part by a postdoctoral fellowship from the National Multiple Sclerosis Society (S.L.F.), National Institutes of Health Grant NS030800 (R.H.M.), and the Myelin Repair Foundation (R.H.M.). We thank Janet Krasno, Becca Lewis, and Anastasia Fete for technical assistance and Stephen Maricich and Anne DeChant for helpful discussions and comments on this manuscript.

Correspondence should be addressed to Robert H. Miller, Department of Neurosciences, Case Western Reserve University School of Medicine, E721, 10900 Euclid Avenue, Cleveland, 0H 44106. E-mail: rhm3@case.edu.

DOI:10.1523/JNEUROSCI.3239-10.2011

Copyright $\odot 2011$ the authors $\quad 0270-6474 / 11 / 310843-08 \$ 15.00 / 0$ al., 1998; Du et al., 2006). Interestingly, exposure of OPCs to PDGF or FGF-2 (Bhat and Zhang, 1996; Yim et al., 2001; Bansal et al., 2003), the neurotrophins NGF, neurotrophin 3, or BDNF (Althaus et al., 1997; Kumar et al., 1998; Du et al., 2006) all activate mitogen-activated protein kinase (MAPK) signaling.

The prototypic members of the MAPK family, Erk1 and Erk2, regulate a diverse array of cellular functions (Rubinfeld and Seger, 2005). Pharmacological inhibition of ERK1/2 activation (pERK1/2) results in fewer oligodendrocytes with mature phenotypes, suggesting a role for the extracellular signal-regulated kinase (ERK) proteins in oligodendrocyte differentiation (Baron et al., 2000; Younes-Rapozo et al., 2009). Similarly, genetic deletion of the upstream ERK regulator B-Raf results in defective oligodendrocyte differentiation (Galabova-Kovacs et al., 2008).

ERK1 and ERK2 exhibit $>80 \%$ sequence homology and have identical substrate specificity (Boulton and Cobb, 1991; Boulton et al., 1991). However, the phenotypes of mice deficient for these genes are quite different (Rubinfeld and Seger, 2005; Aouadi et al., 2006). Erk1 knock-out (Erk1 ${ }^{-/-}$) mice are viable (Mazzucchelli et al., 2002), whereas Erk2 null mice die during embryogenesis (Hatano et al., 2003; Saba-El-Leil et al., 2003; Yao et al., 2003). To date, isoform-specific roles for ERK1 and ERK2 in oligodendrocyte differentiation have not been investigated.

To identify a specific role for ERK2 in oligodendrocyte maturation, Erk2 was deleted from GFAP-expressing $\left(\mathrm{GFAP}^{+}\right)$radial glia that give rise to neurons and oligodendrocytes in the forebrain and from $\mathrm{NG}_{2}{ }^{+} \mathrm{OPCs}$. We show the first in vivo evidence that deletion of Erk2 causes a delay in the appearance of differentiated oligodendrocytes. MBP expression was significantly re- 
duced in the Erk2 conditional knock-out mice (Erk2 CKO) at postnatal day 10 (P10), whereas MBP expression in the Erk1 $1^{-/-}$ mice was indistinguishable from wild-type (WT) littermates. By P21, MBP expression reached WT levels in Erk2 CKO mice, demonstrating that deletion of Erk2 results in a delay but not a complete block of oligodendrocyte differentiation. No deficits in OPC proliferation or total cell number were seen in the Erk2 CKO mice. These findings provide important insights into the role of ERK2 in oligodendrocyte biology.

\section{Materials and Methods}

Primary mixed cultures. Mixed cell cultures were prepared from P5 rat cortex or $\mathrm{P} 3$ mouse cortex and plated on poly-L-lysine-coated coverslips. The media were changed every $2 \mathrm{~d}$. Cells were grown in media consisting of DMEM/ F-12 (Mediatech), $10 \mathrm{ng} / \mathrm{ml}$ PDGF-AA (Sigma), 1\% FBS (HyClone), and N2 supplement. Select cultures were treated with the pERK1/2 inhibitor U0126 [1,4-diamino-2,3-dicyano-1,4-bis(o-aminophenylthio)butadiene] (10 $\mu \mathrm{M}$; Cell Signaling Technology) for $2 \mathrm{~d}$ before immunostaining. PDGF was removed from the P5 rat cortical culture media $2 \mathrm{~d}$ before the addition of U0126.

Purified OPC cultures. Highly enriched populations of immature OPCs were prepared by immunopanning with the monoclonal antibody A2B5 as described previously (Robinson and Miller, 1996). Purified cultures of OPCs from $\mathrm{P} 0$ rat spinal cord were grown in media consisting of DMEM/F-12 (Mediatech), $10 \mathrm{ng} / \mathrm{ml}$ PDGF-AA (Sigma), 1\% FBS (HyClone), and N2 supplement for $1 \mathrm{~d}$. U0126 at $10 \mu \mathrm{M}$ (or DMSO for controls) was added, and cells were incubated in the presence of the drug for $2 \mathrm{~d}$.

Immunocytochemistry of primary cell cultures. Labeling with the monoclonal antibodies A2B5 (1:2), O4 (1:4), and galactocerebroside (GalC) (1:4) was performed on live cells. Cells were incubated in primary antibody diluted in DMEM with 10\% normal goat serum (NGS) for $30 \mathrm{~min}$ followed by secondary antibody (1:500, Alexa Fluor-conjugated mouse IgM). Cells were then postfixed using $5 \%$ acetic acid in methanol. For double labeling with pERK1/2 (1:500; Cell Signaling Technology), yellow fluorescent protein (YFP) [1:500; anti-green fluorescent protein (GFP); Invitrogen], or NG2 (1:500; Millipore Corporation) cells were subsequently incubated in primary antibody for $45 \mathrm{~min}$ in PBST (PBS and $0.3 \%$ Triton X-100) and 10\% NGS, followed by Alexa Fluor-conjugated rabbit IgG and mounted using Vectashield with 4',6-diamidino-2phenylindole (DAPI) (Vector Laboratories).

Bromodeoxyuridine labeling and analysis. To analyze proliferation of cells in S phase, bromodeoxyuridine (BrdU) $(10 \mu \mathrm{M})$ was added to the media of $6 \mathrm{~d}$ in vitro (DIV) cultures $6 \mathrm{~h}$ before fixation. Cells were fixed in $4 \%$ paraformaldehyde for $15 \mathrm{~min}$ and then incubated with anti-NG2 antibody (1:500; Millipore Corporation) in PBST (0.3\% Triton X-100) and $10 \%$ NGS and then Alexa Fluor-conjugated secondary antibody. For double labeling with anti-BrdU, the nuclei were then permeabilized with $2 \mathrm{~N} \mathrm{HCl}$ for $30 \mathrm{~min}$, followed by incubation with anti-BrdU (1:50; Roche) and then Alexa Fluor-conjugated IgG (1:500; Invitrogen).

Cell counts. For purified OPC cultures, the number of marker-positive cells relative to the total number of DAPI-positive cells was counted from six randomly selected fields taken from three different coverslips. For Erk2 CKO cultures, cells were counted from 5 to 10 randomly chosen fields for each coverslip of cells cultured from at least two mice per genotype.

Experimental animals. hGFAP-Cre mice on an FVB/N background (Zhuo et al., 2001) were interbred with Erk2 floxed mice (Samuels et al., 2008), which were homozygous for the Rosa-YFP reporter allele (The Jackson Laboratory) on a C57BL/6 background to generate $h G F A P-$ Cre/+; Erk2 flox/flox; Rosa YFP/+ animals in which the ERK2 gene has been conditionally knocked out. Similarly, NG2-Cre (Cspg4-Cre; The Jackson Laboratory) mice on a mixed C57BL/6 and FVB genetic background were interbred with $\mathrm{C} 57 \mathrm{BL} / 6$ Erk2 floxed mice to create oligodendrocyte-specific NG2--Cre; Erk2 CKO mice. The generation of the Erk1 $1^{-1-}$ mice was described previously (Nekrasova et al., 2005). All mice were kept in microisolation in a pathogen-free environment in the Animal Resource Center of Case Western Reserve University, and all procedures were conducted according to approved Institutional Animal Care and Use Committee guidelines.

Immunostaining of frozen sections. Coronal cryosections $(20 \mu \mathrm{m})$ through the mouse forebrain were rinsed in PBS, blocked, and permeabilized with $0.3 \%$ Triton X-100 and $2 \%$ goat serum in PBS. Sections were incubated with primary antibodies at $4^{\circ} \mathrm{C}$ overnight, washed with $\mathrm{PBS}$, and incubated with secondary antibodies at room temperature for $1 \mathrm{~h}$. The sections were then counterstained with DAPI for $3 \mathrm{~min}$. AntiMBP (1:1000; SMI-99; Covance) or anti-NG2 (1:500; Millipore Corporation) were used for primary antibodies, and Alexa Fluor-conjugated $\operatorname{IgG}$ (1:500; Invitrogen) was used as the secondary antibody. At least 20 sections from two to five mice per genotype were analyzed for each experiment.

Western immunoblotting. Mouse cortex and underlying white matter was dissected on ice and snap frozen in a dry ice/ethanol bath. Each protein sample was collected from one mouse. Samples from four mice per genotype were collected and analyzed for WT and GFAP-Cre; Erk2 CKO mice. Samples from two mice were collected for $\mathrm{Erk1}^{-1-}$ and NG2Cre; Erk2 CKO. Cells were lysed by sonication in radioimmunoprecipitation assay buffer (50 mu Tris, $\mathrm{pH} 8,150 \mathrm{~mm} \mathrm{NaCl}, 1 \% \mathrm{NP}-40,0.5 \%$ sodium deoxycholate, and $0.1 \%$ SDS) supplemented with $1 \times$ protease inhibitor cocktail (Halt Protease and Phosphatase Inhibitor Cocktail; Thermo Fisher Scientific). Lysates were cleared by centrifugation, and protein concentration was determined using the bicinchoninic acid assay (Pierce) with BSA as a standard. Proteins were boiled in sample buffer, separated by SDS-PAGE gels, and transferred to Immobilon-P polyvinylidene difluoride membranes (Millipore Corporation). Membranes were blocked in 5\% skim-milk powder in TBS and $0.2 \%$ Tween 20 (TBS-T) for $1 \mathrm{~h}$ at room temperature and incubated overnight at $4^{\circ} \mathrm{C}$ with primary antibodies. The primary antibodies used were mouse anti-glyceraldehyde-3-phosphate dehydrogenase (GAPDH) (1:10,000; Santa Cruz Biotechnology) and mouse anti-MBP (SMI-99; 1:1000; Covance). After washing with TBS-T, membranes were incubated with HRP-conjugated goat antimouse (1:5000; ICN Biomedicals) or anti-rabbit (1:5000; Cappel) secondary antibodies in TBS-T with $5 \%$ milk for $1 \mathrm{~h}$ at room temperature. Blots were washed with TBS-T and developed with Pierce chemiluminescence reagents. Densitometric analysis was performed using the Adobe Photoshop histogram function.

Electron microscopy. For ultrastructural analyses, P21 mice were perfused with $4 \%$ paraformaldehyde and $2 \%$ glutaraldehyde in $0.1 \mathrm{M} \mathrm{caco-}$ dylate buffer, pH 7.4 (Electron Microscopy Sciences). Tissue was dissected and postfixed in $1 \% \mathrm{OsO}_{4}$. Samples were taken from the cortex and midline of the corpus callosum of Erk2 CKO and WT mice. Samples were dehydrated through graded ethanols, stained en bloc with uranyl acetate, and embedded in a Poly/Bed812 resin (Polysciences Inc.). Thin $(1 \mu \mathrm{m})$ sections were stained with toluidine blue, and ultrathin $(0.1 \mu \mathrm{m})$ sections from matching areas of experimental and control tissue blocks were cut and visualized using an electron microscope (JEOL1200CX) at $80 \mathrm{kV}$. Sections were analyzed from two mutant and two WT mice.

Statistical analysis. Statistical analysis was performed using the Student's $t$ test, as indicated in the figures. In all graphs, the error bars indicate SE.

\section{Results}

Activated ERK1/2 (pERK1/2) is expressed by oligodendrocyte progenitor cells as they differentiate

To verify the expression of pERK1/2 in $\left(\mathrm{O}^{+}\right)$oligodendrocyte progenitors and at the stage where late progenitors are entering terminal differentiation $\left(\mathrm{GalC}^{+}\right)$, colabeling of these markers with pERK1/2 was performed (Fig. $1 A$ ). pERK1/2 was highly expressed in the nucleus of both $\mathrm{O}_{4}{ }^{+}$and $\mathrm{GalC}^{+}$oligodendrocytes demonstrating active signaling through the ERK pathway as pERK1/2 are translocated and retained in the nucleus in which they act to regulate gene expression. 


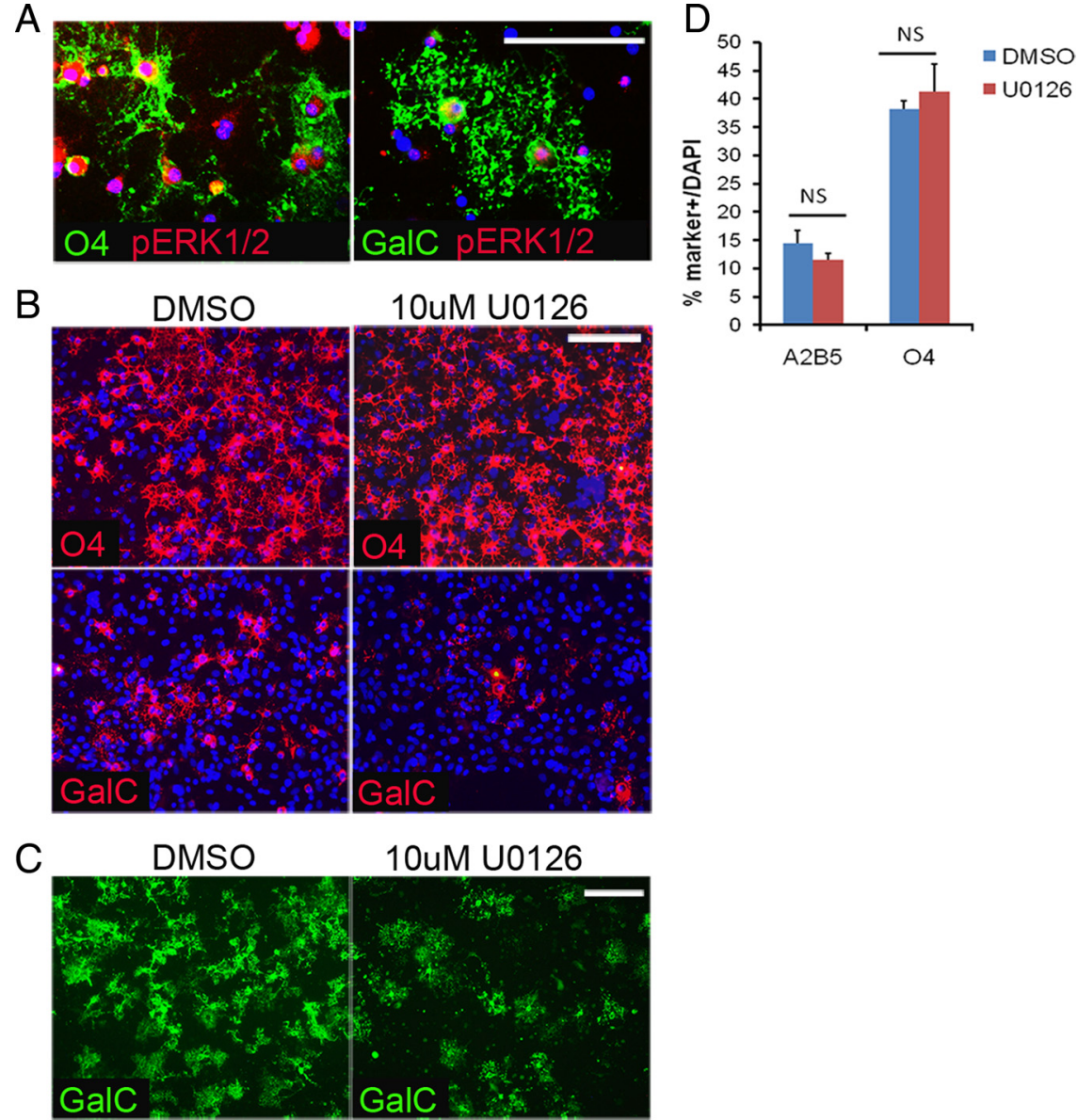

Figure 1. Activated ERK1/2 (pERK1/2) is expressed in both $04^{+}$and $\mathrm{GalC}^{+}$oligodendrocytes and is necessary for the appearance of GalC ${ }^{+}$oligodendrocytes in vitro. $A$, Anti-pERK1/2 (red) and anti-04 (green, left) or anti-GalC (green, right) immunostaining of $\mathrm{A} 2 \mathrm{~B} 5{ }^{+}$pan-purified oligodendrocyte cultures after 5 DIV. B, Anti-04 (red, top) or anti-GalC (red, bottom) immunostaining of mixed cultures from P5 rat brain treated with $10 \mu \mathrm{m}$ U0126 or DMSO. DAPI staining (blue) marks cell nuclei. C, Anti-GaIC (green) immunostaining of $\mathrm{A2B}^{+}{ }^{+}$pan-purified oligodendrocyte cultures from P0 spinal cord treated with $10 \mu \mathrm{m}$ U0126 or DMSO. D, The number of $\mathrm{A2B5}^{+}$or $04^{+} \mathrm{OPCs}$ was quantified and reported as a percentage of the total number of DAPI ${ }^{+}$cells from $\mathrm{A} 2 \mathrm{~B} 5{ }^{+}$ pan-purified cultures treated with $10 \mu \mathrm{m}$ U0126 or DMSO. Scale bars, $100 \mu \mathrm{m}$. NS, Not significant.

\section{Activated forms of ERK are necessary for oligodendrocyte differentiation in vitro}

To determine whether activated ERKs are important for the transition from $\mathrm{O}^{+}{ }^{+} \mathrm{OPC}$ to $\mathrm{GalC}^{+}$oligodendrocytes, their activation was blocked by inhibiting the upstream kinases MEK1/2 by adding U0126 $(10 \mu \mathrm{M})$ to mixed cultures from P5 rat brain after 5 DIV. The majority of the oligodendrocyte lineage cells in these cultures were $\mathrm{O}_{4}{ }^{+}$but GalC-negative at the time of drug administration (data not shown). After $2 \mathrm{~d}$ of $\mathrm{pERK} 1 / 2$ inhibition, there was no difference in the number of $4^{+}$OPCs (Fig. $1 B$, top), but a robust decrease in the number of mature $\mathrm{GalC}^{+}$oligodendrocytes was observed (Fig. $1 B$, bottom), suggesting that ERK1/2 activation is necessary for the differentiation of $\mathrm{O}^{+}$OPCs into mature oligodendrocytes. To determine whether the observed differentiation block was specifically attributable to a direct effect on OPCs, $\mathrm{A} 2 \mathrm{~B} 5{ }^{+}$pan-purified OPCs were cultured from $\mathrm{P} 0$ rat spinal cord and treated with $10 \mu \mathrm{M}$ U0126 after 1 DIV. Cultures were exposed to U0126 for $2 \mathrm{~d}$. Similar to what was observed in mixed culture, the number of $\mathrm{GalC}^{+}$terminally differentiated oligodendrocytes was greatly reduced in purified OPC cultures after pERK1/2 was blocked with U0126 ( $8 \pm 1 \%$ with DMSO vs $2 \pm 1 \%$ with U0126; $p=0.007$ ) (Fig. $1 C$ ). Both the percentage of $\mathrm{A}_{2} 5^{+}$early OPCs $(14 \pm 2 \%$ with DMSO vs $11 \pm 1 \%$ with
$\mathrm{U} 0126 ; p>0.05)$ and $\mathrm{O}^{+}$late OPCs $(38 \pm 1 \%$ with DMSO vs $41 \pm 5 \%$ with U0126; $p>0.05$ ) was not significantly different between U0126 treated and DMSO control cultures (Fig. 1D), suggesting that pERK1/2 is important for oligodendrocyte differentiation but not for the survival or proliferation of early OPCs.

\section{OPC differentiation is impaired in mixed primary cortical cultures from Erk2 CKO mice}

The roles of the ERKs in OPC development have not been examined previously in vivo because Erk2 null animals are not viable. To define the individual role of ERK2, Cre-LoxP technology was used to conditionally delete Erk2 from GFAP ${ }^{+}$ progenitor cells in the mouse. The $h G F A P-C r e$ transgene is expressed starting at embryonic day 13.5 (E13.5) in cells that give rise to neurons, astrocytes and oligodendrocytes (Zhuo et al., 2001). Fate mapping studies have demonstrated that $\sim 67 \%$ of all oligodendrocytes in the corpus callosum are derived from $\mathrm{GFAP}^{+}$ progenitor cells (Casper and McCarthy, 2006). To create Erk2 CKO mice, $h$ GFAPCre mice (Zhuo et al., 2001) were crossed with mice that carry an Erk2 allele flanked by LoxP sites (Erk2 flox) as well as a RosaYFP reporter allele (stock \#006148; The Jackson Laboratory). A detailed analysis of the generation of the Erk2 flox mice and of neural development in hGFAP-Cre; Erk2 flox/flox (Erk2 CKO) mice has been described previously (Samuels et al., 2008).

To determine whether deletion of Erk2 alone would lead to an inhibition in oligodendrocyte differentiation similar to that seen after blocking both pERK1 and pERK2 with the U0126 inhibitor, mixed cultures from P3 Erk2 CKO mouse cortex were grown for 12-13 DIV. In these experiments, $\mathrm{YFP}^{+}$cells have undergone Cre-mediated recombination, resulting in the deletion of Erk2 in the Erk2 CKO cultures. In Erk2 flox/ + control cultures, there is one WT allele and one mutant allele so the $\mathrm{YFP}^{+}$cells express Erk2 from their WT allele. Significantly fewer mature double-labeled $\mathrm{YFP}^{+}$, $\mathrm{GalC}^{+}$oligodendrocytes were observed in the Erk2 CKO cultures $(75 \pm 4 \%)$ compared with cultures from heterozygous control littermates $(93 \pm 3 \%$ ) (Fig. $2 A-C$ ), demonstrating that ERK2 is important for oligodendrocyte differentiation.

Because ERK1 and ERK2 are very similar proteins that are able to phosphorylate identical substrates, ERK1 has been postulated to compensate for the loss of ERK2 (Samuels et al., 2008). To determine whether ERK1 is playing an important role in enabling the Erk2 CKO oligodendrocytes to progress to the $\mathrm{GalC}^{+}$stage, we treated Erk2 CKO mixed cortical cultures with U0126 to block pERK1 activity at 10 DIV. By 12 DIV, there was a $48 \%$ decrease in the number of $\mathrm{GalC}^{+}$mature oligodendrocytes after pERK1 was blocked in Erk2 CKO cultures (Erk2 flox/+, $93 \pm 3 \%$; Erk2 CKO, $75 \pm 4 \%$; Erk2 CKO + U0126, $26 \pm 6 \%$ ) (Fig. 2C). These data suggest that the deletion of Erk2 dramatically reduces the number 
of mature oligodendrocytes in vitro and that, in the absence of ERK2, ERK1 is able to compensate enabling a subset of Erk2 CKO OPCs to differentiate.

The decrease in the number of mature oligodendrocytes in cultures from Erk2 CKO cortex is not a result of reduced proliferation or a reduction in the number of oligodendrocyte progenitors The decreased numbers of $\mathrm{GalC}^{+}$oligodendrocytes observed in the Erk2 CKO cultures could be attributable to a block in differentiation or a reduction in the proliferation and number of OPCs. To investigate these possibilities, the number of $\mathrm{NG} 2{ }^{+} \mathrm{OPCs}$ was assessed. The total number of $\mathrm{NG}^{+}$, $\mathrm{YFP}^{+}$cells was not significantly different between Erk2 CKO cultures and cultures from heterozygous control littermates after 12 DIV (Erk2 flox/+, $81 \pm 26$; Erk2 CKO, $62 \pm 16 ; p>0.05)$, suggesting that there is not a deficit in the number of OPCs available to go on to become mature myelinating cells. To assess whether the proliferation of OPCs was affected by the deletion of Erk2, the thymidine analog BrdU was added to the cell growth medium of mixed cortical cultures. The percentage of proliferating $\mathrm{NG}_{2}{ }^{+}, \mathrm{BrdU}^{+}$oligodendrocyte lineage cells $\left(\mathrm{NG}^{+}{ }^{+}, \mathrm{BrdU}^{+} / \mathrm{NG}^{+}{ }^{+}\right.$) was not different between Erk2 CKO and control cultures (Erk2 flox/+, $76 \pm 3 \%$; Erk2 CKO, $69 \pm 3 \% ; p>0.05)$, suggesting that ERK2 is not required for the proliferation of OPCs.

\section{ERK2 plays a critical role in the timing of} forebrain myelination

To determine whether ERK2 is necessary for the maturation of oligodendrocytes in vivo, the expression of the mature oligodendrocyte marker MBP was evaluated in P10 Erk2 CKO mice. MBP levels were clearly decreased in the corpus callosum of Erk2 CKO mice compared with WT controls (Fig. 3A). MBP expression was also analyzed in $\mathrm{rrk1}^{-/-}$(Nekrasova et al., 2005) corpus callosum to determine whether ERK1 may play an important role in oligodendrocyte differentiation. In contrast to the data from the Erk2 $\mathrm{CKO}$ animals, the absence of ERK1 alone does not appear to affect oligodendrocyte differentiation because MBP expression in $E r k 1^{-1-}$ corpus callosum is comparable with WT levels at P10 (Fig. 3A). To quantify the expression changes in the Erk2 CKO mice, proteins were isolated from the cortex and corpus callosum at P10, and Western blots were probed for MBP and GAPDH as a loading control (Fig. 3B). Consistent with the immunofluorescence data, MBP levels were significantly decreased in Erk2 CKO samples but were comparable with WT levels in the Erk1 $1^{-1-}$ mice.

To determine whether the deletion of Erk2 affects the number of OPCs in vivo, NG2 expression was monitored in the corpus callosum of P2 mice. Similar to the observations in vitro, no difference in the number of $\mathrm{NG}^{+}{ }^{+}$cells was detected between Erk2 CKO mice and littermate controls, suggesting that ERK2 is not important for the early specification or proliferation of OPCs (Fig. 3C). Interestingly, the decreased levels
YFP
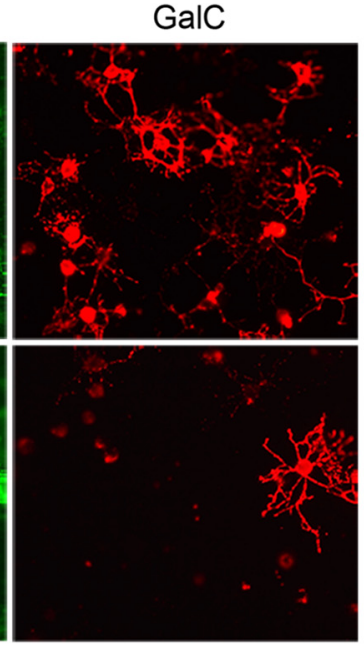

C
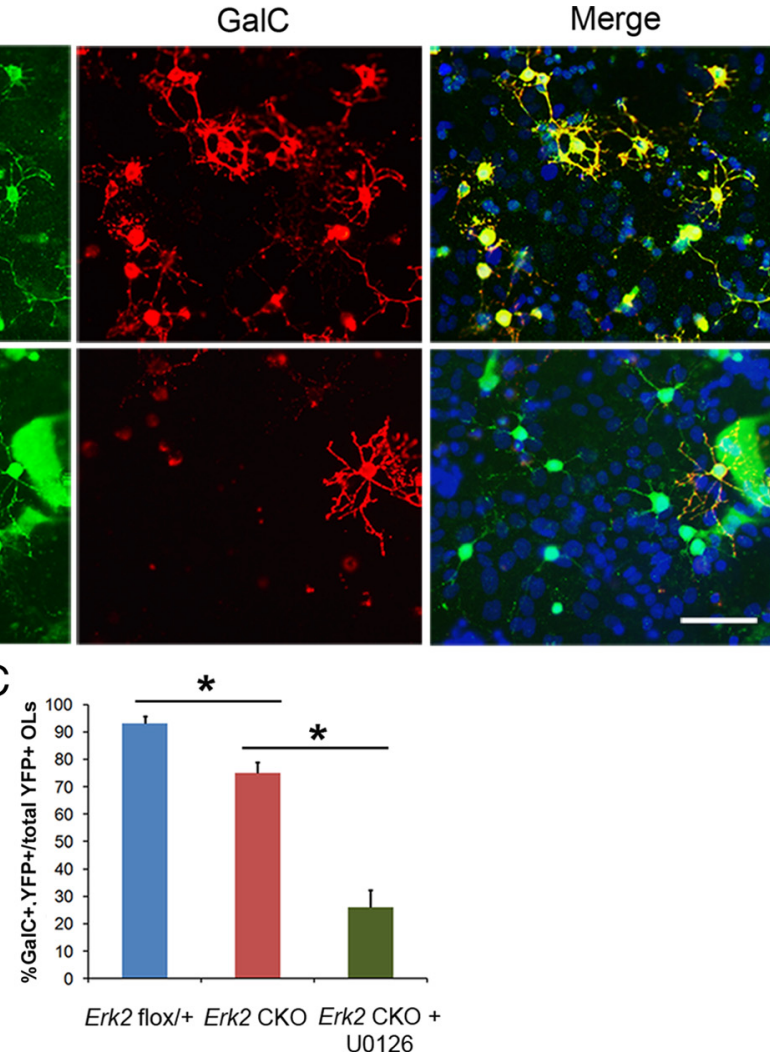

Figure 2. OPC differentiation is impaired in cortical cultures from GFAP-Cre; Erk2 CKO mice. $\boldsymbol{A}, \boldsymbol{B}$, Anti-GFP (green) and antiGalC (red) immunostaining of mixed cultures from Erk2 flox/+ (A) and Erk2 CKO (B) P3 cortex after 12 DIV. C, The number of lineage cells. U0126 at $10 \mu \mathrm{m}$ was added to a subset of the Erk2 CKO cultures to block ERK1 activity $2 \mathrm{~d}$ before the cells were analyzed by immunostaining. Scale bar, $100 \mu \mathrm{m} .{ }^{*} p<0.001$, unpaired $t$ test.

of MBP expression found in the Erk2 CKO corpus callosum appear to be transient because more normal MBP expression is observed by P21 (Fig. 4A). Furthermore, a more detailed analysis of the corpus callosum from the midline of Erk2 CKO mice and control littermates at P21 demonstrated a mild residual hypomyelinated phenotype. Although myelination was relatively uniform across the corpus callosum in WT animals, it appeared quite patchy in the Erk2 CKO animals. Some areas had a density of myelinated fibers that was indistinguishable from WT corpus callosum, whereas adjacent areas showed mild hypomyelination (Fig. $4 B$ ). Ultrastructurally, however, the myelin that was formed appeared normal (Fig. 4C). Together, these data clearly implicate ERK2 in the regulation of OPC differentiation but not in the induction, proliferation, or survival of OPCs.

Because the $h G F A P$ promoter drives Cre expression in radial glia starting at E13.5 resulting in the permanent deletion of Erk2 from astrocytes, neurons, and oligodendrocytes, it is possible that deletion of Erk2 from several different cell types contributes to the myelin phenotype early in development. To gain insight into the cellular specificity of the ERK2 activity required for the proper timing of forebrain myelination, we expressed Cre recombinase specifically in $\mathrm{NG} 2{ }^{+}$oligodendrocyte progenitor cells (NG2-Cre) to delete Erk2 from these cells. MBP expression appeared to be decreased in the corpus callosum of P7 NG2-Cre; Erk2 CKO mice compared with control littermates (Fig. $5 A, B$ ) but was restored to WT levels by P21 (Fig. $5 C$ ). These data suggest that a cell-autonomous defect exists within 

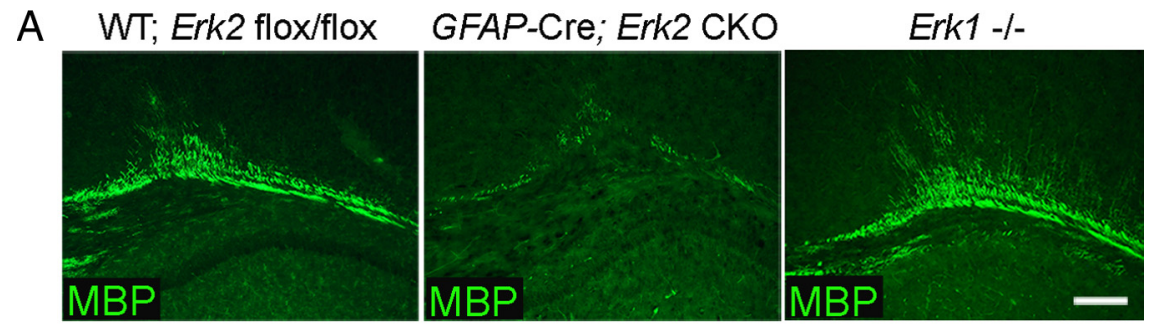

B

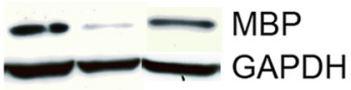

WT Erk2 Erk1-/$\mathrm{CKO}$

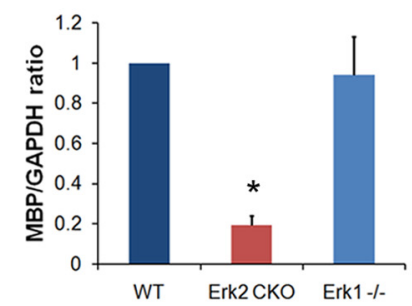

C WT; Erk2 flox/flox
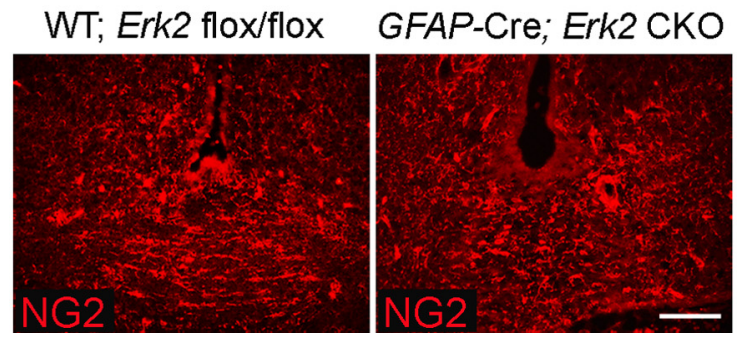

Figure 3. Selective requirement for ERK2 in the timing of forebrain myelination. $A$, Anti-MBP immunostaining (green) of corpus callosum from GFAP-Cre; Erk2 CKO, Erk ${ }^{-1-}$, and control littermate mice at P10. B, Western blot analysis and quantification of MBP from P10 cortical lysates. GAPDH levels serve as a loading control. C, Anti-NG2 immunostaining (red) of corpus callosum from P2 mice. Scale bars, $100 \mu \mathrm{m} .{ }^{*} p<0.05$, unpaired $t$ test.

oligodendrocytes lacking ERK2, which interferes with their ability to differentiate.

\section{Discussion}

Our data demonstrate that ERK2 is critical for the proper timing of the appearance of differentiated oligodendrocytes in vivo. Specifically, after genetic ablation of Erk2, OPCs were stalled at the late progenitor stage with a delayed ability to progress to $\mathrm{GalC}^{+}$ or $\mathrm{MBP}^{+}$mature oligodendrocytes. In contrast, Erk1 ${ }^{-/-}$OPCs appeared to transition to mature oligodendrocytes similar to control littermates, suggesting that ERK1 does not normally play an important role in oligodendrocyte maturation. In the absence of ERK2, however, our in vitro experiments demonstrate that ERK1 is able to compensate enabling a subset of Erk2 CKO OPCs to differentiate. Finally, we demonstrate that both the proliferation and the survival of $\mathrm{NG}^{+}$OPCs appears to be unaffected in the Erk2 CKO mouse cortex.

The dependence on ERK2 for oligodendrocyte differentiation does not appear to be absolute because $75 \%$ of the oligodendrocytes that had lost ERK2 expression $\left(\mathrm{YFP}^{+}\right)$were still able to progress to the mature $\mathrm{GalC}^{+}$stage (Fig. $2 \mathrm{C}$ ). Because these cells were cultured for $12 \mathrm{DIV}$, it is likely that, over time, another protein is able to compensate for the loss of ERK2, thereby enabling a number of OPCs to progress from $\mathrm{O}^{+}{ }^{+}$to $\mathrm{GalC}^{+}$differentiated oligodendrocytes. The in vivo data presented here support this hypothesis because the differentiation impairment observed in OPCs from the Erk2 CKO corpus callosum is transient. MBP expression was essentially restored to WT levels in the Erk2 CKO mice by P21, and the mice have normal lifespans without any obvious physical abnormalities suggestive of a myelin deficiency. Furthermore, blocking pERK1 in Erk2 CKO cultures resulted in far fewer $\mathrm{GalC}^{+}$oligodendrocytes, suggesting that pERK1 is likely compensating for the loss of ERK2 under these culture conditions. Because of the complex nature of the oligodendrocyte differentiation process, it may be that several other proteins in addition to ERK1 have the ability to compensate for the lack of ERK2 over time. For example, mTOR (the mammalian target of rapamycin), which is a member of the phosphatidylinositol 3-kinase/Akt signaling pathway and is known to be important for oligodendrocyte differentiation and myelination (Narayanan et al., 2009; Tyler et al., 2009), may also have the ability to compensate for the loss of ERK2 through the upregulation of this parallel pathway, and this might contribute to the transient phenotype of the Erk2 CKO mice. Interestingly, not all myelinated regions are equally affected by the lack of ERK2 because, in hindbrain regions such as the cerebellum, MBP levels appear close to WT at P10 (data not shown). This may reflect the fact that, during normal development, oligodendrocyte differentiation progresses in a caudal-to-rostral gradient. Therefore, a careful examination of MBP expression in hindbrain regions at earlier time points might detect a transient defect.

Another possible explanation for the recovery of MBP expression by $\mathrm{P} 21$ in the Erk2 CKO mice is that, over time, Erk2 $2^{-\prime-}$ OPCs that were born in the medial ganglionic eminence (MGE) or the lateral ganglionic eminence (LGE) and subsequently migrated into the cortex may have been replaced by WT OPCs derived from the postnatal cortex. Because the $h G F A P-C r e$ is only expressed in astrocytes in the postnatal period, OPCs born postnatally from progenitor cells that have never expressed GFAP would not undergo recombination and Erk2 would not be deleted. Consistent with this hypothesis is the residual patches of hypomyelination in the animals at P21. Cre-LoxP fate mapping has demonstrated that there are three waves of OPC generation in the mouse forebrain (Kessaris et al., 2006). The first wave arises from the MGE at E12.5, the second arises from the LGE at E15.5, and the third arises locally in the cortex starting after birth. Importantly, by P10, there are very few MGE-derived OPCs left in the cortex. The LGE and cortically derived OPC populations appear to take over as the mice mature. After $12 \mathrm{DIV}$, comparable numbers of YFP ${ }^{+}$ OPCs are found in both Erk2 CKO and control cultures (Fig. 2D), demonstrating that at least at this time point Cre-mediated recombination has occurred and Erk2 has been deleted from these cortical OPCs. It is, however, possible that, as these mice age, the pool of Erk2 $2^{-/-}$OPCs is slowly replaced by WT cells throughout the cortex.

The $h$ GFAP promoter drives Cre expression in radial glia starting at E13.5, resulting in the permanent deletion of Erk2 from astrocytes, neurons, and oligodendrocytes. It was therefore important to determine whether aberrant signaling from mutant neurons, astrocytes, or oligodendrocytes was responsible for the defects in oligodendrocyte differentiation observed in the Erk2 CKO mice. To determine whether ERK2 


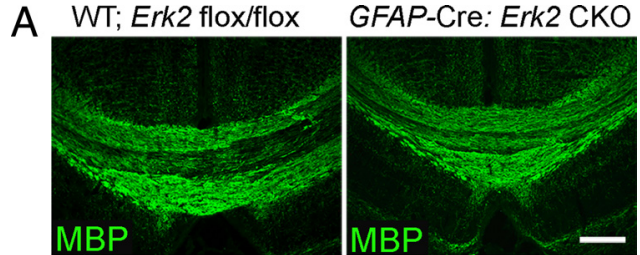

B WT; Erk2 flox/flox

GFAP-Cre: Erk2 CKO

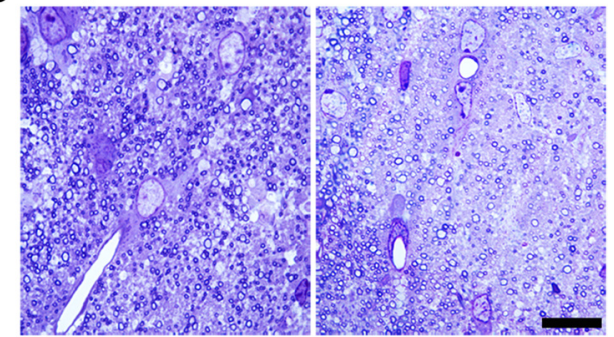

C

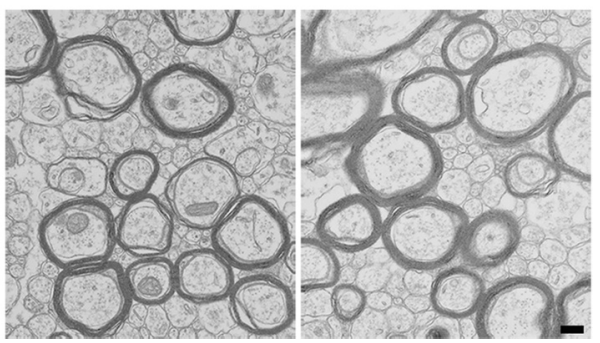

Figure 4. The myelination phenotype in GFAP-Cre; Erk2 CKO mice appears transient. $\boldsymbol{A}$ Anti-MBP immunostaining (green) of corpus callosum from P21 Erk2 CKO and control littermate. $\boldsymbol{B}$, Toluidine blue staining demonstrates that Erk2 CKO animals retain a patchy hypomyelination phenotype in the midline corpus callosum at P21. WT animals have a relatively uniform distribution of myelinated axons, whereas Erk2 CKO animals have areas of normal myelination interspersed with areas of hypomyelination. $C$, The ultrastructure of the myelin was similar between WT and Erk2 CK0 animals at P21. Scale bars: $A, 100 \mu \mathrm{m}$; $B, 15 \mu \mathrm{m} ; C, 200 \mathrm{~nm}$.

activity within oligodendrocyte lineage cells was required for the proper timing of forebrain myelination, Erk2 was specifically deleted from $\mathrm{NG}_{2}{ }^{+}$OPCs. MBP expression was decreased in the corpus callosum of NG2-Cre; Erk2 CKO mice at P7, demonstrating an oligodendrocyte-specific effect of Erk2 deletion on the timing of forebrain myelination. These results are consistent with the data presented in Figure $1 C$ in which inhibition of pERK1/2 in purified cultures of OPCs was sufficient to cause a significant block in oligodendrocyte differentiation. It is important to note, however, that the decrease in MBP staining observed in the NG2-Cre; Erk2 CKO corpus callosum was less robust than that seen in the GFAP-Cre; Erk2 CKO mice, suggesting that, although ERK2 expression within OPCs is clearly important for the proper timing of myelination, the lack of ERK2 in adjacent neurons and/or astrocytes may contribute to the delayed myelination phenotype. Previous work has demonstrated that ERK2 plays a critical role in neural development in the developing cortex. Loss of ERK2 from GFAP ${ }^{+}$progenitor cells results in impaired proliferation of neural progenitors, leading to a $10 \%$ reduction in the number of neuronal-specific nuclear protein-expressing cortical neurons per region analyzed and profound deficits in associative learning (Samuels et al., 2008). Because neurons participate in the development of oligodendrocytes by regulating their proliferation, differentiation, and survival (Barres and Raff, 1999), it is possible that aberrant signals from ERK2-deficient neurons could contribute to the defects in oligodendrocyte differentiation observed in this
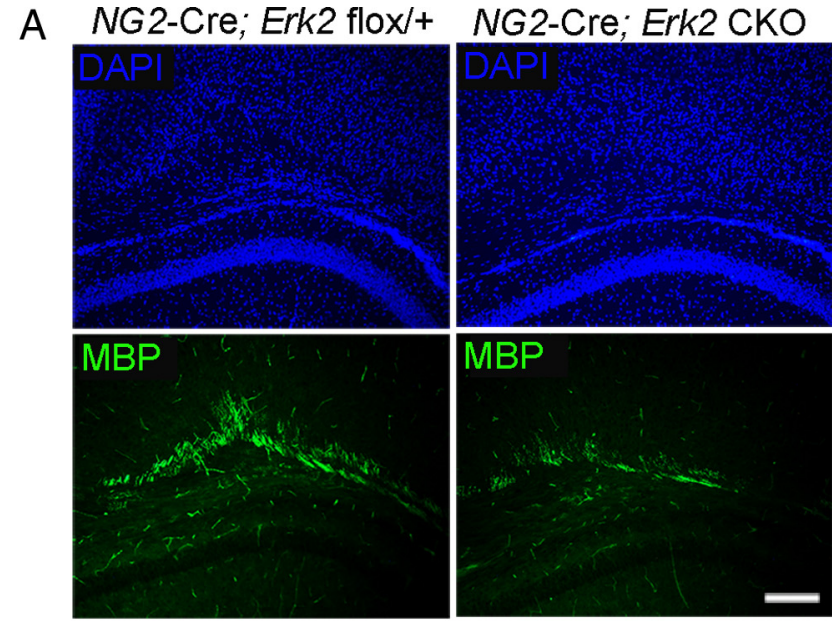

B
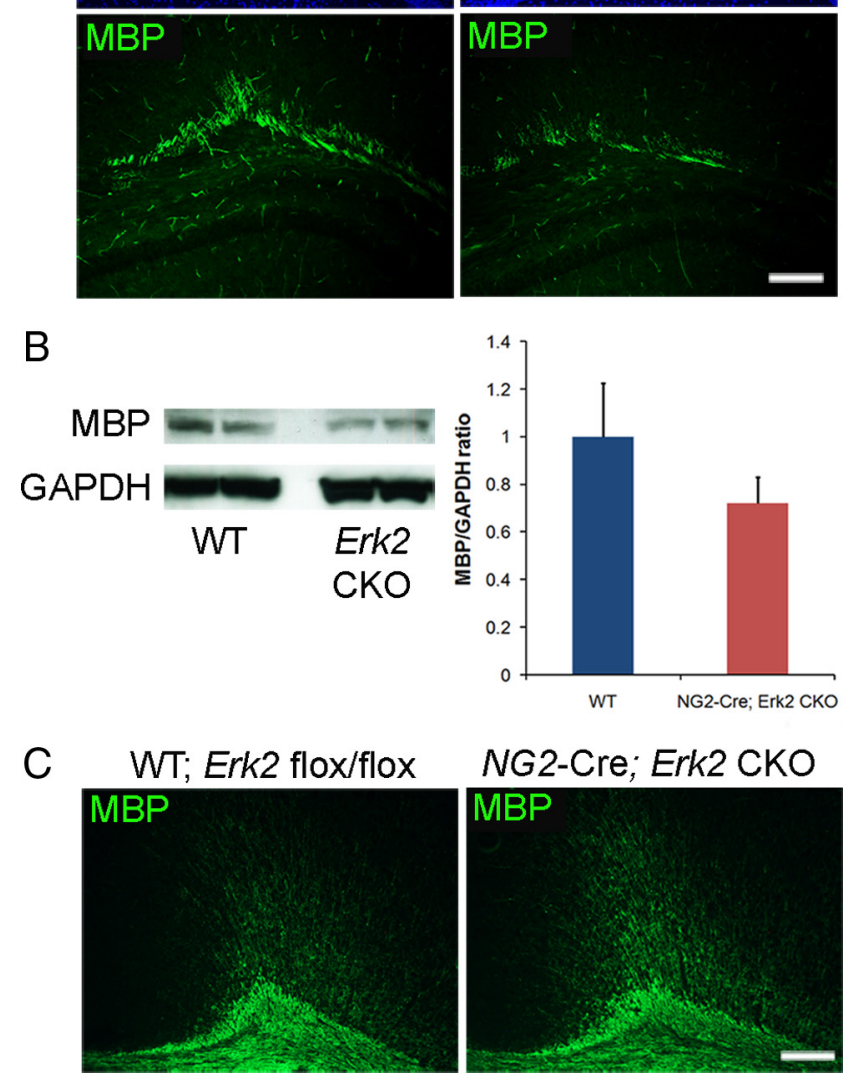

NG2-Cre; Erk2 CKO MBP

Figure 5. ERK2 expression in oligodendrocyte progenitor cells is important for the proper timing of forebrain myelination. A, Anti-MBP immunostaining (green) of corpus callosum from NG2-Cre; Erk2 CKO and control littermate at P7. DAPI staining marks cell nuclei. $\boldsymbol{B}$, Western blot analysis and quantification of MBP from P7 cortical lysates demonstrates a trend toward decreased levels of MBP in the NG2-Cre; Erk2 CKO mice. $n=2$ animals per genotype. GAPDH levels serve as a loading control. C, Anti-MBP immunostaining (green) of corpus callosum from NG2-Cre; Erk2 CK0 mice and littermate control at P21. Scale bars, $100 \mu \mathrm{m}$

study. Likewise, because astrocytes secrete soluble factors that are oligodendrocyte supportive (Arai and Lo, 2010), it is also possible that ERK2-deficient astrocytes could contribute to the observed defects in oligodendrocyte differentiation.

We have demonstrated that ERK2 is necessary for the transition from OPC to mature oligodendrocyte, but it remains unknown whether ERK2 is sufficient to drive the differentiation of OPCs. An interesting extension of these studies would be to evaluate whether the overexpression of constitutively active ERK2 variants can enhance myelination by pushing more OPCs to transition to mature oligodendrocytes. Latestage OPCs can be found in and around demyelinated lesions in multiple sclerosis patients, suggesting that remyelination by adult OPCs is not inhibited as a result of a lack of mobility or recruitment but rather by an inability of these cells to terminally differentiate (Scolding et al., 1998; Wolswijk, 1998; Chang et al., 2002; Kuhlmann et al., 2008). Adenovirus infec- 
tion of oligodendrocytes with constitutively active variants of the ERK1/2 upstream kinase MAP kinase kinase (MEK) has been shown to result in significant increases in MBP expression in vitro (Du et al., 2006). Future experiments will address whether high levels of ERK2 may be sufficient to drive oligodendrocyte differentiation in OPCs that are retained as immature cells in and around demyelinated lesions, potentially providing a new direction for the design of therapeutic approaches to facilitate myelin repair.

An important question that remains unanswered in the field of oligodendrocyte biology is what determines whether an individual OPC goes on to become a myelinating oligodendrocyte versus one that remains in an undifferentiated state (Chong and Chan, 2010). Adult OPCs account for $\sim 2-9 \%$ of the CNS population (Dawson et al., 2003) and may represent an important reservoir of cells that can contribute to remyelination in the diseased CNS. It remains unclear how these cells differ from the majority of the OPCs that differentiate into mature myelinating oligodendrocytes during development. It is tempting to speculate that ERK2 may be an important regulator of the genesis of adult OPCs. Future studies will address whether careful titration of the ERK2 protein or activation levels may provide a mechanism to control the ability of an individual OPC to differentiate. A better understanding of inductive factors important for oligodendrocyte differentiation and CNS myelination should aid in the identification of therapeutic targets to promote remyelination in the diseased CNS.

\section{References}

Althaus HH, Hempel R, Klöppner S, Engel J, Schmidt-Schultz T, Kruska L, Heumann R (1997) Nerve growth factor signal transduction in mature pig oligodendrocytes. J Neurosci Res 50:729-742.

Aouadi M, Binetruy B, Caron L, Le Marchand-Brustel Y, Bost F (2006) Role of MAPKs in development and differentiation: lessons from knockout mice. Biochimie 88:1091-1098.

Arai K, Lo EH (2010) Astrocytes protect oligodendrocyte precursor cells via MEK/ERK and PI3K/Akt signaling. J Neurosci Res 88:758-763.

Bansal R, Pfeiffer SE (1994) Inhibition of protein and lipid sulfation in oligodendrocytes blocks biological responses to FGF-2 and retards cytoarchitectural maturation, but not developmental lineage progression. Dev Biol 162:511-524.

Bansal R, Magge S, Winkler S (2003) Specific inhibitor of FGF receptor signaling: FGF-2-mediated effects on proliferation, differentiation, and MAPK activation are inhibited by PD173074 in oligodendrocyte-lineage cells. J Neurosci Res 74:486-493.

Baron W, Metz B, Bansal R, Hoekstra D, de Vries H (2000) PDGF and FGF-2 signaling in oligodendrocyte progenitor cells: regulation of proliferation and differentiation by multiple intracellular signaling pathways. Mol Cell Neurosci 15:314-329.

Barres BA, Raff MC (1999) Axonal control of oligodendrocyte development. J Cell Biol 147:1123-1128.

Barres BA, Hart IK, Coles HS, Burne JF, Voyvodic JT, Richardson WD, Raff MC (1992) Cell death and control of cell survival in the oligodendrocyte lineage. Cell 70:31-46.

Bhat NR, Zhang P (1996) Activation of mitogen-activated protein kinases in oligodendrocytes. J Neurochem 66:1986-1994.

Boulton TG, Cobb MH (1991) Identification of multiple extracellular signal-regulated kinases (ERKs) with antipeptide antibodies. Cell Regul 2:357-371.

Boulton TG, Nye SH, Robbins DJ, Ip NY, Radziejewska E, Morgenbesser SD, DePinho RA, Panayotatos N, Cobb MH, Yancopoulos GD (1991) ERKs: a family of protein-serine/threonine kinases that are activated and tyrosine phosphorylated in response to insulin and NGF. Cell 65:663-675.

Casper KB, McCarthy KD (2006) GFAP-positive progenitor cells produce neurons and oligodendrocytes throughout the CNS. Mol Cell Neurosci 31:676-684.
Chang A, Tourtellotte WW, Rudick R, Trapp BD (2002) Premyelinating oligodendrocytes in chronic lesions of multiple sclerosis. N Engl J Med 346:165-173.

Chong SY, Chan JR (2010) Tapping into the glial reservoir: cells committed to remaining uncommitted. J Cell Biol 188:305-312.

Dawson MR, Polito A, Levine JM, Reynolds R (2003) NG2-expressing glial progenitor cells: an abundant and widespread population of cycling cells in the adult rat CNS. Mol Cell Neurosci 24:476-488.

Du Y, Lercher LD, Zhou R, Dreyfus CF (2006) Mitogen-activated protein kinase pathway mediates effects of brain-derived neurotrophic factor on differentiation of basal forebrain oligodendrocytes. J Neurosci Res 84:1692-1702.

Galabova-Kovacs G, Catalanotti F, Matzen D, Reyes GX, Zezula J, Herbst R, Silva A, Walter I, Baccarini M (2008) Essential role of B-Raf in oligodendrocyte maturation and myelination during postnatal central nervous system development. J Cell Biol 180:947-955.

Hatano N, Mori Y, Oh-hora M, Kosugi A, Fujikawa T, Nakai N, Niwa H, Miyazaki J, Hamaoka T, Ogata M (2003) Essential role for ERK2 mitogen-activated protein kinase in placental development. Genes Cells 8:847-856.

Kessaris N, Fogarty M, Iannarelli P, Grist M, Wegner M, Richardson WD (2006) Competing waves of oligodendrocytes in the forebrain and postnatal elimination of an embryonic lineage. Nat Neurosci 9:173-179.

Kuhlmann T, Miron V, Cui Q, Wegner C, Antel J, Brück W (2008) Differentiation block of oligodendroglial progenitor cells as a cause for remyelination failure in chronic multiple sclerosis. Brain [Erratum (2009) 132: 1118 (Cuo Q corrected to Cui Q)] 131:1749-1758.

Kumar S, Kahn MA, Dinh L, de Vellis J (1998) NT-3-mediated TrkC receptor activation promotes proliferation and cell survival of rodent progenitor oligodendrocyte cells in vitro and in vivo. J Neurosci Res 54:754-765.

Mazzucchelli C, Vantaggiato C, Ciamei A, Fasano S, Pakhotin P, Krezel W, Welzl H, Wolfer DP, Pagès G, Valverde O, Marowsky A, Porrazzo A, Orban PC, Maldonado R, Ehrengruber MU, Cestari V, Lipp HP, Chapman PF, Pouysségur J, Brambilla R (2002) Knockout of ERK1 MAP kinase enhances synaptic plasticity in the striatum and facilitates striatal-mediated learning and memory. Neuron 34:807-820.

McKinnon RD, Matsui T, Dubois-Dalcq M, Aaronson SA (1990) FGF modulates the PDGF-driven pathway of oligodendrocyte development. Neuron 5:603-614.

Miller RH (1996) Oligodendrocyte origins. Trends Neurosci 19:92-96.

Mozell RL, McMorris FA (1991) Insulin-like growth factor I stimulates oligodendrocyte development and myelination in rat brain aggregate cultures. J Neurosci Res 30:382-390.

Narayanan SP, Flores AI, Wang F, Macklin WB (2009) Akt signals through the mammalian target of rapamycin pathway to regulate CNS myelination. J Neurosci 29:6860-6870.

Nekrasova T, Shive C, Gao Y, Kawamura K, Guardia R, Landreth G, Forsthuber TG (2005) ERK1-deficient mice show normal T cell effector function and are highly susceptible to experimental autoimmune encephalomyelitis. J Immunol 175:2374-2380.

Noble M, Murray K, Stroobant P, Waterfield MD, Riddle P (1988) Plateletderived growth factor promotes division and motility and inhibits premature differentiation of the oligodendrocyte/type-2 astrocyte progenitor cell. Nature 333:560-562.

Pfeiffer SE, Warrington AE, Bansal R (1993) The oligodendrocyte and its many cellular processes. Trends Cell Biol 3:191-197.

Raff MC, Lillien LE, Richardson WD, Burne JF, Noble MD (1988) Plateletderived growth factor from astrocytes drives the clock that times oligodendrocyte development in culture. Nature 333:562-565.

Robinson S, Miller R (1996) Environmental enhancement of growth factormediated oligodendrocyte precursor proliferation. Mol Cell Neurosci $8: 38-52$.

Rubinfeld H, Seger R (2005) The ERK cascade: a prototype of MAPK signaling. Mol Biotechnol 31:151-174.

Saba-El-Leil MK, Vella FD, Vernay B, Voisin L, Chen L, Labrecque N, Ang SL, Meloche S (2003) An essential function of the mitogen-activated protein kinase Erk2 in mouse trophoblast development. EMBO Rep 4:964-968.

Samuels IS, Karlo JC, Faruzzi AN, Pickering K, Herrup K, Sweatt JD, Saitta SC, Landreth GE (2008) Deletion of ERK2 mitogen-activated protein 
kinase identifies its key roles in cortical neurogenesis and cognitive function. J Neurosci 28:6983-6995.

Scolding N, Franklin R, Stevens S, Heldin CH, Compston A, Newcombe J (1998) Oligodendrocyte progenitors are present in the normal adult human CNS and in the lesions of multiple sclerosis. Brain 121:22212228.

Tyler WA, Gangoli N, Gokina P, Kim HA, Covey M, Levison SW, Wood TL (2009) Activation of the mammalian target of rapamycin (mTOR) is essential for oligodendrocyte differentiation. J Neurosci 29:6367-6378.

Wolswijk G (1998) Chronic stage multiple sclerosis lesions contain a relatively quiescent population of oligodendrocyte precursor cells. J Neurosci 18:601-609.

Yao Y, Li W, Wu J, Germann UA, Su MS, Kuida K, Boucher DM (2003)
Extracellular signal-regulated kinase 2 is necessary for mesoderm differentiation. Proc Natl Acad Sci U S A 100:12759-12764.

Yim SH, Hammer JA, Quarles RH (2001) Differences in signal transduction pathways by which platelet-derived and fibroblast growth factors activate extracellular signal-regulated kinase in differentiating oligodendrocytes. J Neurochem 76:1925-1934.

Younes-Rapozo V, Felgueiras LO, Viana NL, Fierro IM, Barja-Fidalgo C, Manhães AC, Barradas PC (2009) A role for the MAPK/ERK pathway in oligodendroglial differentiation in vitro: stage specific effects on cell branching. Int J Dev Neurosci 27:757-768.

Zhuo L, Theis M, Alvarez-Maya I, Brenner M, Willecke K, Messing A (2001) hGFAP-cre transgenic mice for manipulation of glial and neuronal function in vivo. Genesis 31:85-94. 